\title{
Dexamethasone Resisted Podocyte Injury via Stabilizing TRPC6 Expression and Distribution
}

\author{
Shengyou Yu and L. Yu \\ Guangzhou Medical College, Guangzhou First Municipal People's Hospital, Guangdong Province, Guangzhou 510180, China
}

Correspondence should be addressed to L. Yu, yul828@yeah.net

Received 23 July 2011; Accepted 19 January 2012

Academic Editor: Esra Küpeli Akkol

Copyright () 2012 S. Yu and L. Yu. This is an open access article distributed under the Creative Commons Attribution License, which permits unrestricted use, distribution, and reproduction in any medium, provided the original work is properly cited.

TRPC6, a member of the canonical transient receptor potential channel (TRPC) subfamily, is an important cation selective ion channel on podocytes. Podocytes are highly differentiated cells located on the visceral face of glomerular basement membrane and featured by numerous foot processes, on which nephrin, podocin, and TRPC6 locate. Podocytes and the slit diaphragm (SD) between adjacent foot processes form a selective filtration barrier impermeable to proteins. TRPC6 is very critical for normal podocyte function. To investigate the function of TRPC6 in podocytes and its relation to proteinuria in kidney diseases, we overexpressed TRPC6 in podocytes by puromycin aminonucleoside (PAN) and observed the changes of foot processes, TRPC6 protein distribution, and mRNA expression. Accordingly, in this study, we further investigated the role of specific signaling mechanisms underlying the prosurvival effects of dexamethasone (DEX) on podocyte repair. Our results showed that podocytes processes of overexpressing TRPC6 were reduced remarkably. These changes could be rescued by DEX via blocking TRPC6 channel. Additionally, our results also showed an improvement in TRPC6 arrangement in the cells and decrease of mRNA expression and protein distribution. From these results, we therefore proposed that overexpression of TRPC6 in podocytes may be one of the fundamental changes relating to the dysfunction of the SD and proteinuria. DEX may be maintained the structure and function integrity of SD by blocking TRPC6 signal pathway and played an important role in mechanisms of anti-proteinuria.

\section{Introduction}

TRPC6 is determined in recent years which is closely related with proteinuria and positioned in the structure protein molecules of SD. Proteinuria is a common feature of kidney dysfunction of glomerular origin and is itself a risk factor for renal disease [1]. There is a growing body of experimental and clinical literature showing that podocyte number is a critical determinant for the development of proteinuria and glomerulosclerosis [2]. PAN is used to induce a wellestablished cell model for podocytes injury. DEX is widely used for the treatment of a variety of glomerular diseases characterized by podocyte injury and proteinuria, including membranous nephropathy, minimal change disease, FSGS, and lupus nephritis. However, the signaling mechanisms underlying the antiproteinuria effects of DEX have not been well defined. In this study, we further investigated the role of specific signaling mechanisms underlying the protection effects of DEX on podocyte injury. Our results showed that, in podocytes overexpressing TRPC6, cell processes were reduced remarkably. These changes could be rescued by the treatment of the cells with DEX to block TRPC6 channel. Additionally, the podocytes overexpressing TRPC6 treated with DEX showed an improvement in TRPC6 arrangement in the cells and decrease of mRNA expression and protein distribution.

\section{Materials and Methods}

2.1. Cells in Culture. Conditionally immortalized mouse podocyte clone (a kindly gift from Professor Peter Mundel, USA, and Professor Jie DING, Peking University First Hospital) was cultured at $33^{\circ} \mathrm{C}$ in RPMI- 1640 containing $10 \%$ fetal bovine serum (Gibco, Gaithersburg, MD, USA), $100 \mathrm{U} / \mathrm{mL}$ penicillin/streptomycin, and $10 \mathrm{U} / \mathrm{mL}$ of mouse recombinant r-interferon (PEPRO Tech, London, UK) and then shifted to $37^{\circ} \mathrm{C}$ for differentiation by removal of $\mathrm{r}$ interferon [3]. had typical character of mature podocyte after 
two weeks. In the studies described below, all experiments were performed in growth-restricted podocytes.

2.2. Experimental Design. In order to examine the effect of DEX on PAN-induced podocyte injury, podocytes grown under growth restrictive conditions for 12 days were incubated with media containing $10 \%$ FBS in the presence of $1 \mu \mathrm{mol} / \mathrm{L}$ DEX (Sigma Chemical Co). DEX was not removed until the end of each experiment. To determine if DEX reduced a range of injuries, the following experiments were undertaken after $1 \mathrm{~h}$ of DEX incubation: PAN. To determine the mechanisms of podocyte injury induced by PAN, we exposed podocytes to PAN (Sigma Chemical Co) at a concentration of $50 \mu \mathrm{g} / \mathrm{mL}$. Following a $48 \mathrm{~h}$ incubation with PAN in the presence or absence of DEX, then observed and harvested at $8 \mathrm{~h}, 24 \mathrm{~h}$, and $48 \mathrm{~h}$, respectively. The experiments were all repeated three times.

2.3. RT-PCR Analysis. Total RNA was extracted from podocyte using Trizol reagent according to the manufacturer's instruction, and the RNA concentration was determined after the sample was dissolved in diethylpyrocarbonate-treated water. Isolated RNA $(1 \mu \mathrm{g})$ of each sample was subjected to reverse transcription by using Rever Tra Ace (TOYOBO Co., Japan) according to the manufacture's protocol. The resulting cDNA $(3 \mu \mathrm{L})$ was used for PCR amplification. The sequence-specific primers were designed and synthesized by Shanghai Invitrogen Biotechnology Co, Ltd. Primers used were as follows. TRPC6 upstream and downstream primers, respectively, were as follows. Forward: 5 GTTAATTGCGATGATCAATAGTT-3. Reverse: 5-GACTTGGTACAAGATTGAAGG-3. Probe: 5FAM-CCAGGAAATTGAGGATGATGCGGACGTG-BHQ1-

3 , product length being $143 \mathrm{bp}$. GAPDH upstream and downstream primers were as follows. Forward: 5-GGTG AAGGTCGGTGTGAACGGAT-3. Reverse: 5-CCACTTTGC CACTGCAAATGGCAG-3. Probe: FAM-CTGGTGACCAG GCGCCCAATACGGCC-BHQ1, product length being $118 \mathrm{bp}$. The PCR amplification was started with $2 \mathrm{~min}$ of denaturation at $94^{\circ} \mathrm{C}$, which was followed by 34 cycles (for GAPDH, 30 cycles) of denaturation at $94^{\circ} \mathrm{C}$ for $30 \mathrm{~s}$, annealing at $55^{\circ} \mathrm{C}$ for $30 \mathrm{~s}\left(\mathrm{GAPDH}, 55^{\circ} \mathrm{C}\right.$ for $\left.30 \mathrm{~s}\right)$, and polymerization at $72^{\circ} \mathrm{C}$ for $75 \mathrm{~s}(\mathrm{GAPDH}, 30 \mathrm{~s})$. The final extension lasted $7 \mathrm{~min}$ at $72^{\circ} \mathrm{C}$ and then ended at $4^{\circ} \mathrm{C}$. PCR products $(5 \mu \mathrm{L})$ were separated on $1 \%$ ethidium bromidestained agarose gels and later scanned with gel imaging system (Bio Rad Company A). Independent experiment was repeated 3 times.

2.4. Western Blot Analysis. Podocytes were lyzed in the buffer containing 1\% Tritonx-100, $150 \mathrm{mM} \mathrm{NaCl}, 1 \mathrm{mM}$ EDTA, $50 \mathrm{mM}$ Tris- $\mathrm{HCl}$ ( $\mathrm{pH} 7.7$ ), $1 \mathrm{mM} \mathrm{NaF}, 1 \mathrm{mM} \mathrm{NaVO} 3$, and a protease inhibitor cocktail (Sigma Chemical Co). Seventyfive micrograms of total protein was loaded to run $8 \%$ sodium dodecyl sulfate-polyacrylamide gel electrophoresis (SDS-PAGE), and the gel was set up for transfer protein to nitrocellulose membranes (Sigma Chemical Co.). Then, the membranes were rinsed in a Tris-buffered saline with
0.02\% Tween-20 (TTBS), followed by immersing in 5\% lowfat milk. Subsequently, the membranes were incubated with rabbit anti-TRPC6 antibody (Sigma Chemical Co); mouse anti-GAPDH antibody (Sigma Chemical Co). After rinsing three times with TTBS, the membranes were incubated with HRP-conjugated goat anti-rabbit or mouse IgG (Sigma Chemical Co.) for $45 \mathrm{~min}$ at room temperature and then developed using ECL chemiluminescence reagent (Sigma Chemical Co.). The specific protein bands were scanned and quantitated using densitometry in relation to the GAPDH, Western Blotting Detection System (GE Healthcare, Chalfont St. Giles, UK). We repeated each Western Blot analysis using protein from three different and separate experiments.

2.5. Immunostaining. TRPC6 was fixed with $4 \%$ paraformaldehyde, then permeabilized, and blocked with $0.3 \%$ TritonX-100 and 5\% bovine serum albumin. The primary antibody, rabbit anti-TRPC6 antibody (Sigma Chemical Co), was applied for overnight at $4^{\circ} \mathrm{C}$. FITC-conjugated goat antirabbit or mouse IgG (Sigma Chemical Co) and the nuclei dye Hoechst were used for $45 \mathrm{~min}$ at room temperature. Finally, the coverslips were mounted and images were taken by using a immunofluorescence microscope (Zeiss, Germany). To determine the percentage of the cells in which RPC6 is localized in nuclei, we counted at least 200 nuclei in triplicate in each experiment.

2.6. Statistical Analysis. Data were reported as mean $\pm \mathrm{SD}$ with $\mathrm{n}$ equal to the number of experiments. Statistical evaluation was performed using a one-way ANOVA (twosided test), followed by LSD (equal variances assumed) or Dunnett's T3 (equal variances not assumed) for post hoc test between two groups, and also using the nonparametric tests (Mann-Whitney $U$-test) as a posttest. Values of $P<0.05$ were considered as statistically significance.

\section{Results}

3.1. Effect of DEX on PAN-Induced Podocytes Changes. Podocytes were observed and photographed under inverted microscope. The relative area of podocytes was calculated by Image J and SPSS 13.0. The cell bodies and nucleus of PAN-induced podocytes were significantly decreased. The cell bodies, which connected to each other between cells, stretched out like the branches. Foot processes appeared retraction, and the area of PAN-inducted podocytes was significantly reduced to $75 \%$ at $8 \mathrm{~h}(P<0.05)$; foot processes was obviously retracted, and the cell bodies were reduced to $46 \%$ at $24 \mathrm{~h}(P<0.01)$; shrinked to $27 \%(P<0.01)$, part of foot processes occurred disappearance or lost at $48 \mathrm{~h}$. However, after DEX treated, the area of podocytes was significantly greater at $8 \mathrm{~h}, 24 \mathrm{~h}$, and $48 \mathrm{~h}$, the difference was significant $(P<0.05)$. Based on these findings, we developed the hypothesis that PAN-induced injury maybe prevented by DEX. Figure 1.

3.2. Effect of DEX on Pan-Induced TRPC6 mRNA Expression. TRPC6 mRNA results showed that the target bands can be 


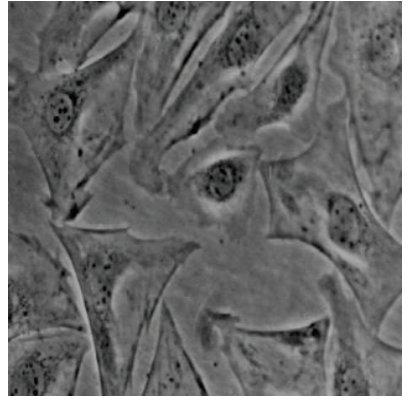

(a)

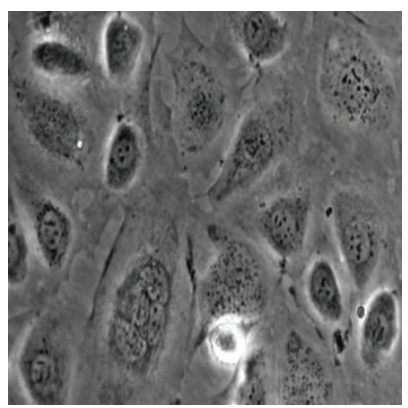

(d)

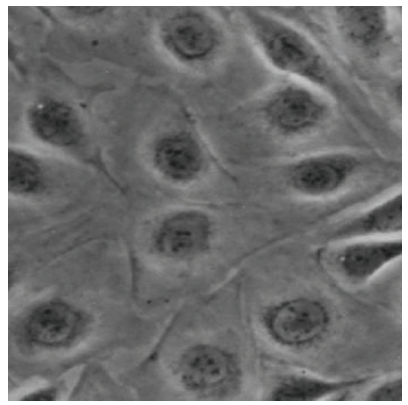

(g)

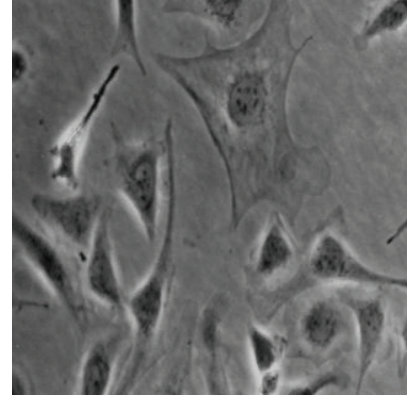

(b)

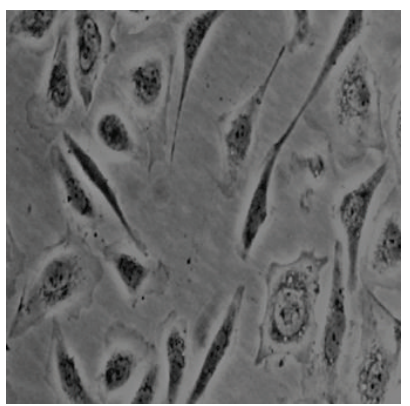

(e)

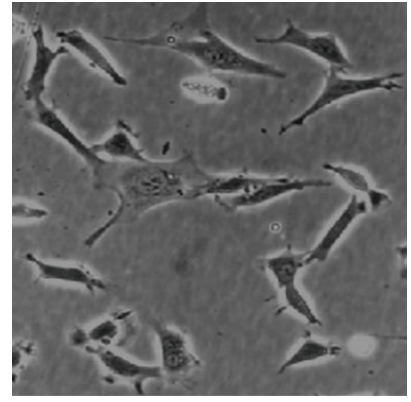

(h)

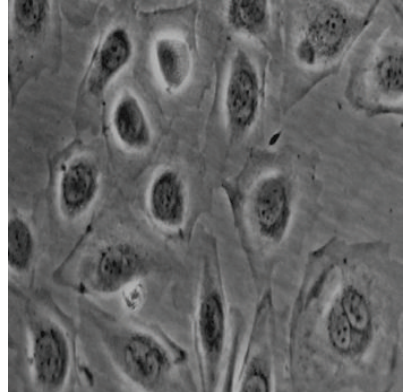

(c)

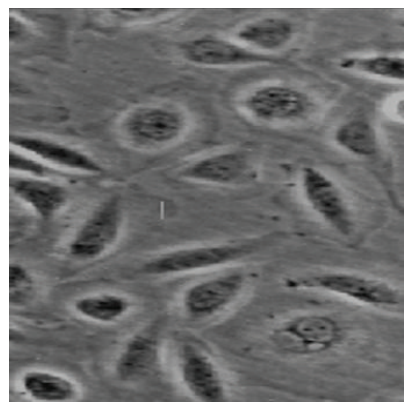

(f)

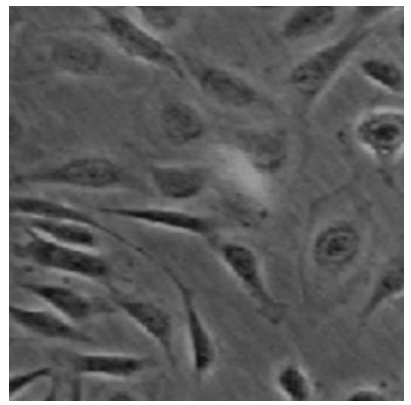

(i)

FIGURE 1: PA-induced podocytes changes at different time points (inverted-phase contrast microscope $\times 200)$. Note: $(a, d, g)$ : the control group, $8 \mathrm{~h}, 24 \mathrm{~h}, 48 \mathrm{~h}$, resp., (foot processes and the connection between podocytes are intact) (b): PAN-induced $8 \mathrm{~h}$; (c): DEX-treated $8 \mathrm{~h}$; (e): PAN-induced $24 \mathrm{~h}$; (f): DEX-treated $24 \mathrm{~h}$; (h): PAN-induced $48 \mathrm{~h}$ (foot process retracted and lost, cell interconnected disappeared); (i): DEX-treated $48 \mathrm{~h}$ (foot processes and the connection between podocytes are still preserved).

seen in the $3 \mathrm{rd}$ and 4 th lanes (150 bp). Our studies showed that there was Trpc6 mRNA expression in the 3rd and 4th samples, but the expression is low, and suggested that TRPC6 mRNA expression is very low under normal circumstances and cannot be shown by the agarose gel electrophoresis. TRPC6 mRNA expression did not change significantly at PAN-induced $8 \mathrm{~h}$ and $24 \mathrm{~h}$. But TRPC6 mRNA expression significantly increased at $48 \mathrm{~h}(P<0.01)$; TRPC6 mRNA expression did not show significantly changes at DEX-treated $8 \mathrm{~h}$; the TRPC6 mRNA expression was significantly decreased at $24 \mathrm{~h}$ and $48 \mathrm{~h}(P<0.01)$. Figure $2(\mathrm{a})$.

Under normal circumstances, TRPC6 expression is very low in podocytes. GAPDH as internal control, Compared with the control group, found that TRPC6 mRNA expression was not significantly changed after PAN-induced $8 \mathrm{~h}$. But TRPC6 mRNA expression slightly increased at $24 \mathrm{~h}$ and $48 \mathrm{~h}$
$(P<0.05)$; TRPC6 mRNA expression significantly decreased after DEX-treated $8 \mathrm{~h}, 24 \mathrm{~h}$, and $48 \mathrm{~h}(P<0.05)$, Figures 2(b) and $2(\mathrm{c})$.

3.3. Effect of DEX on PA-Induced TRPC6 Protein Expression. Western Blot analysis showed that TRPC6 and GAPDH, respectively, have specific band at $36 \mathrm{kd}$ and $106 \mathrm{kd}$. Under normal circumstances, podocytes have a certain amount of TRPC6 protein expression. Compared with the control, TRPC6 protein expression did not change significantly at PAN-induced $8 \mathrm{~h}$ but was higher at $24 \mathrm{~h}$ and $48 \mathrm{~h}(P<0.01)$; TRPC6 protein expression did not significantly change at DEX-treated $8 \mathrm{~h}$; decreased at $24 \mathrm{~h}$; became normal $(P<$ $0.05)$. The protein expression significantly decreased at $48 \mathrm{~h}$ $(P<0.01)$, Figures $3(\mathrm{a})$ and $3(\mathrm{~b})$. 


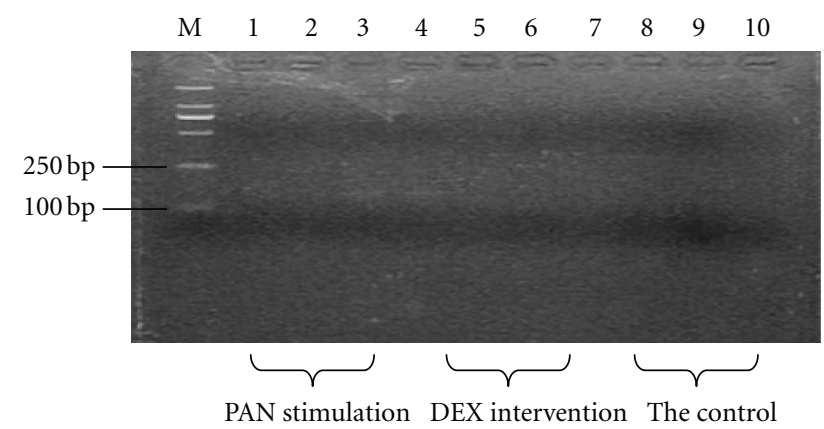

(a)

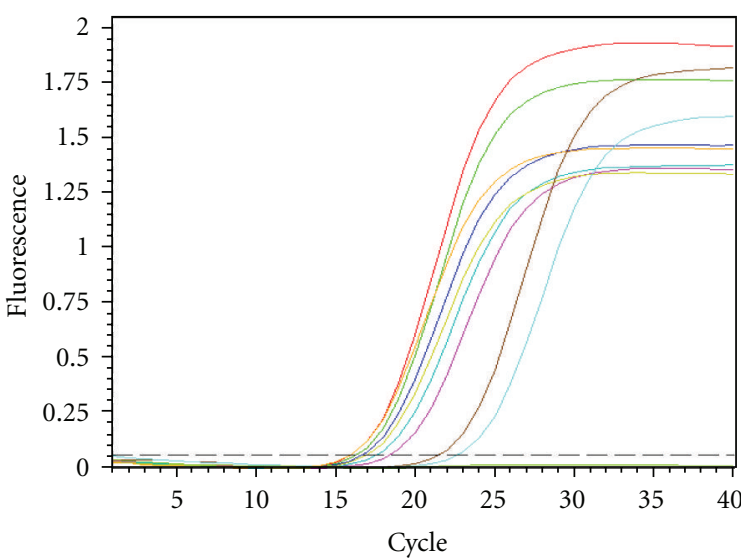

(b)

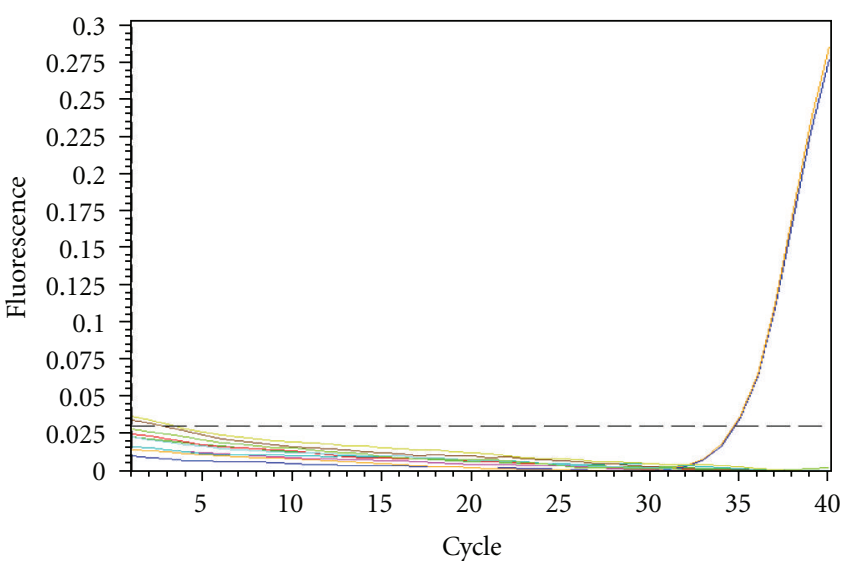

(c)

FIGURE 2: (a) TRPC6 mRNA expression changes at different time points. Note: 1, 4, 7-8 h; 2, 5, 8-24 h; 3, 6, 9-48 h. (b) GAPDH amplification map. (c) TRPC6 amplification map. Note: there was a slight peak in 3rd and 4th samples, ct value reached 34.77, 34.69, indicated that there was Trpc6 mRNA expression in the 3rd and 4th samples, but the expression is low.

\subsection{Effect of DEX on PAN-Induced TRPC6 Distribution} Changes in Podocytes. TRPC6 was linear and evenly distributed in the control, little in the cytoplasm; at PANinducted $24 \mathrm{~h}$, TRPC6 was not continuity distribution along the cell membrane, increased in cytoplasm; at $48 \mathrm{~h}$, TRPC6 distribution increased in cell membrane, part of the cell membrane lost or gathered into granular, widely distributed in cytoplasm. But, after DEX-treated, TRPC6, which significantly improved at different time points, is more homogeneous distribution in the plasma membrane and became normal. Figure 4.

\section{Discussion}

TRPC6 is a member of the large transient receptor potential superfamily of nonselective cation channels $[4,5]$. This superfamily consists of a group of six transmembrane domain-containing ion channels and has been subdivided into six subfamilies; TRPC3; TRPC6, and TRPC7 have about $75 \%$ homology, together constitute heteromeric polymer, and form the function channel [6]. Firstly, Winn et al. [7] and Reiser et al. [2], in 2005, determined one of the pathogenic genes of causing familial focal segmental glomerulosclerosis (FSGS). Further studies showed that there was interaction between TRPC6 protein and SD molecules, together constituting the diaphragm hole complex. This finding will make podocyte structural proteins and ion channels linked. Recent studies have also shown that TRPC6 is expressed in foot processes. TRPC6 within the cell body of podocytes and in primary processes in close vicinity to $\mathrm{SD}$ was associated with the nephrin, podocin, and CD2AP [8]. TRPC6 is a component of the SD multiprotein complex, involved in the physiologic and pathophysiologic role of podocytes [9].

Glucocorticoids are widely used for the treatment of glomerular diseases characterized by podocyte injury and proteinuria [10]. It has been speculated that glucocorticoids exert therapeutic effects through their immunosuppressive and anti-inflammatory mechanisms [11]. Foot processes and SD contribute to the formation of the glomerular filter. It plays an important role in maintaining the podocyte function. Podocyte injury may result in a leakage of proteins into the urine. So injury to podocytes and their SD typically leads to marked proteinuria. PAN, a podocyte toxin, is wildly used to induce experimental nephrotic syndrome in rats [1214], In in vitro study, PAN results in foot process effacement and variable dose-dependent apoptosis [15]. As a new SD molecule, TRPC6 is an important molecule for keeping the structure and function of podocytes as well as regulation of signaling transduction in podocytes and interacting with 


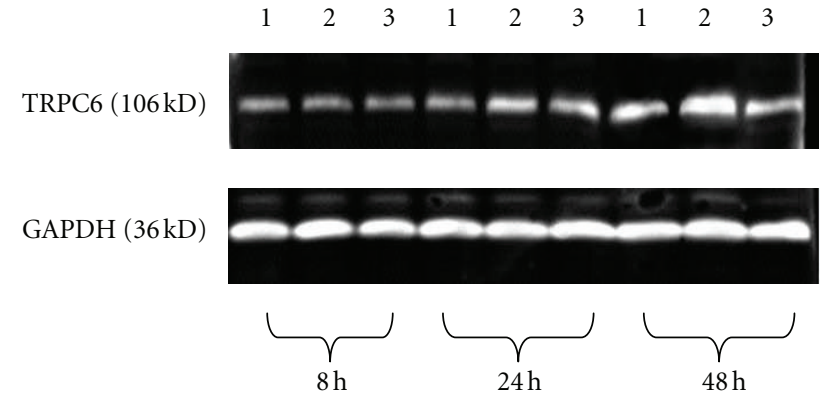

(a)

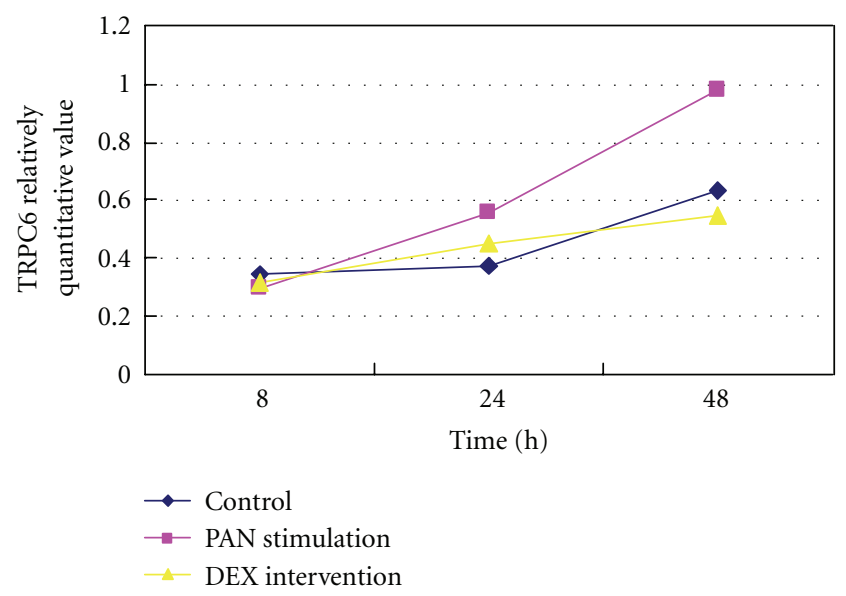

(b)

Figure 3: (a) The Western Blot band of TRPC6 and GAPDH at different time points. Note: (1) the control; (2) PAN stimulation; (3) DEX intervention. (b) The Western Blot analysis of TRPC6 and GAPDH at each time point. Note: the Western Blot analysis showed that the protein level of TRPC6 was increased at PAN-induced $24 \mathrm{~h}$ and $48 \mathrm{~h}$, the difference was significant, $P<0.01$; the protein level of TRPC6 was decreased at DEX-treated $24 \mathrm{~h}$ and $48 \mathrm{~h}$, the difference was significant, $P<0.05$.

other SD molecules, such as nephrin, podocin, and CD2AP [16-18]. Reiser et al. found that TRPC6 was expressed at the podocyte membrane. In nongenetic forms of glomerular disease, such as minimal change disease (NCD), membranous glomerulonephritis (MGN), and FSGS, TRPC6 overexpresion can directly affect cytoskeletal organization in podocytes [19]. Möller et al. also observed that TRPC6 overexpression correlated with the development of podocyte injury. All of these suggest that TRPC6 overexpression is a pivotal factor resulting in podocyte injury. In the PAN-injected rats, the TRPC6 levels were increased markedly [19]. Knocking-down TRPC6 could effectively prevent the podocytes apoptosis induced by PAN [12]. PAN results in foot process effacement and variable dose-dependent apoptosis [20, 21]. On the basis of these findings, we hypothesized that TRPC6 would also take part in the PAN-induced podocyte injury. DEX resisted podocyte injury via stabilizing TRPC6 expression and distribution. The major purpose of our study was to determine whether DEX mediated the specific cytoprotective effect by blocking TRPC6-signaling pathway and blocking TRPC6 channels maybe beneficial to protect podocytes from injury.

Our study found that, at PAN-induced and DEX-treated $8 \mathrm{~h}$, TRPC6 had no significant changes in protein expression. but was significantly higher at PAN-inducted $24 \mathrm{~h}$ and $48 \mathrm{~h}$ $(P<0.01)$; TRPC6 protein expression was significantly decreased at DEX-treated $24 \mathrm{~h}$ and $48 \mathrm{~h}(P<0.05)$. Immunofluorescence staining suggested that TRPC6 showed a linear uniformly distribution in the control group, only a little in the cytoplasm; at PAN-inducted 24h, TRPC6 was mainly punctate distribution along the membrane and increased in cytoplasmic. At $48 \mathrm{~h}$, TRPC6 distribution increased in the cell membrane, part lost, gathered into a granular, widely distributed in the cytoplasm; TRPC6 was more homogeneous distribution after DEX treatment. The distribution was significantly weaker in cytoplasm. The difference, which is compared with other podocyte molecules, was that TRPC6 expression strengthened in PANinducted podocytes, the distribution increased but reduced after DEX-treated. This is the special position of TRPC6 as a cation channel protein. But the specific mechanism is not very clear, pending further study. Our results suggested that TRPC6 might be a pharmacological target of maintaining the function of podocytes in the future. So blocking TRPC6 channels may be a new therapeutic strategy in proteinuric renal diseases.

Our evidence also suggests that blocking TRPC6 channels may be of therapeutic benefit in proteinuria. This creates the exciting possibility that blocking TRPC6 channels within the podocyte may translate into long-lasting clinical benefits in patients with FSGS. Our study suggested that TRPC6 filled the damage signal, which was induced by PAN, and then activated the downstream molecules, caused the injury signal transduction, so that foot process responds to retracted and lost. TRPC6 abnormal expression and distribution led to imbalance of TRPC6 channels and function. The changes of podocyte filtration rate caused the occurrence of proteinuria; the DEX may have made the downstream molecules, which have been activated, inactivated, podocytes restored their original structure by blocking TRPC6 signal channels, thus maintained SD structural and functional integrity by stabilizing TRPC6 expression, and played a protective role in anti-proteinuria.

In summary, TRPC6 overexpression may be one of the fundamental changes in podocytes leading to proteinuria and impairment of renal function. Perhaps, blocking the TRPC6 channel may be candidate for diagnosis and therapeutic target of renal diseases. Our study suggested that TRPC6 signal pathway participated in the signal transduction mechanisms of DEX inhibiting podocyte injury induced by PAN. DEX stabilized TRPC6 expression through binding to its receptor. Our study will provide more clearly theoretical basis for the molecular mechanism of DEX antiproteinuria and also provide new insights into the special beneficial effects of DEX on podocyte injury. 


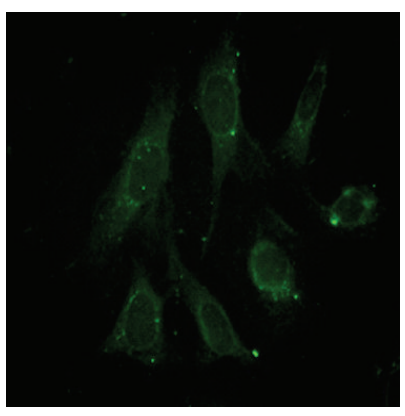

(a)

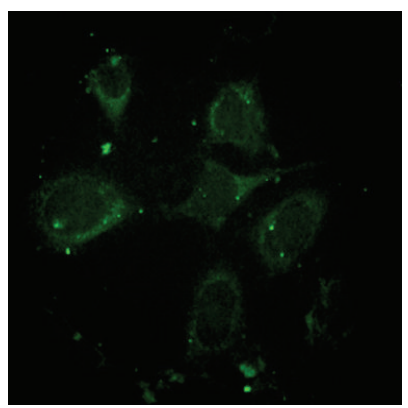

(d)

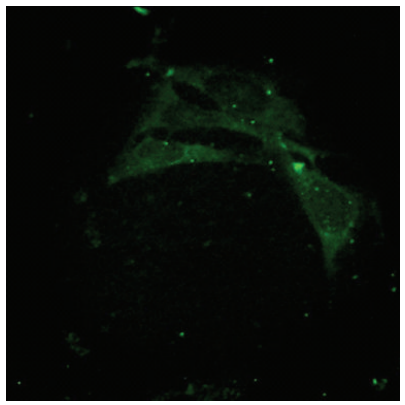

(g)

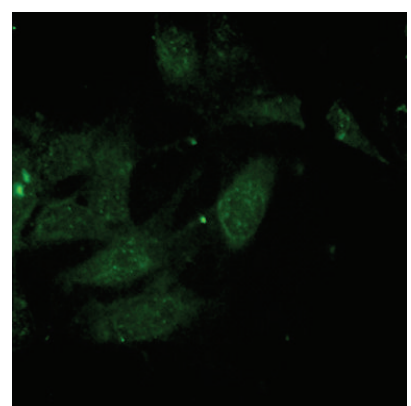

(b)

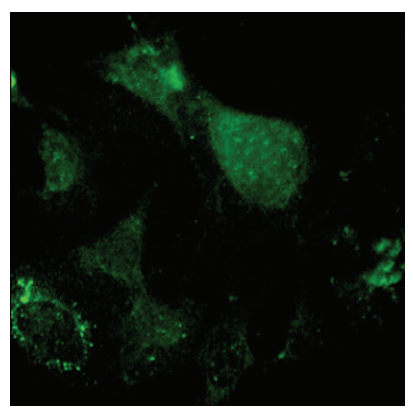

(e)

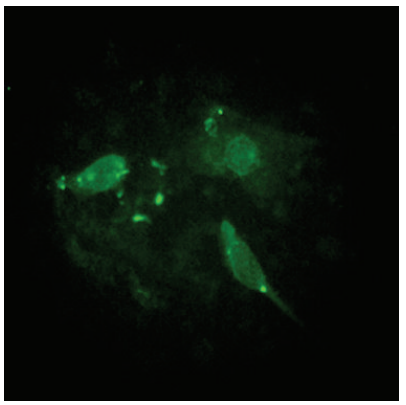

(h)

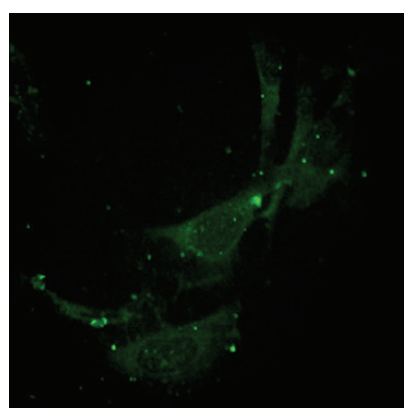

(c)

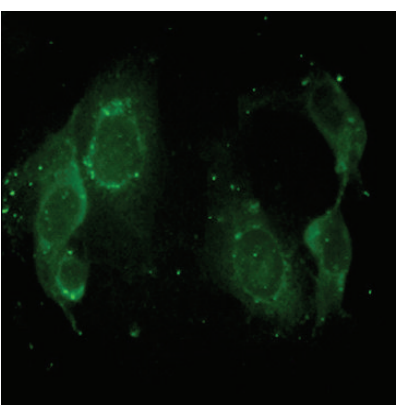

(f)

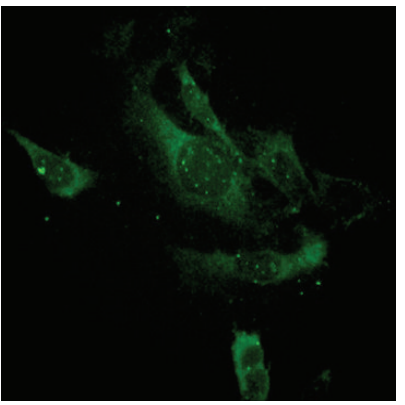

(i)

FIGURE 4: Effects of DEX and PAN on the distribution and protein expression of TRPC6 at different time points (fluorescencemicroscope $\times 400$ ). Note: ( $, \mathrm{d}, \mathrm{g})$ were the control group, TRPC6 was linear and evenly distributed in the podocytes membrane surface, there are some distribution in the cytoplasm; (b) PAN-inducted $8 \mathrm{~h}$; (e) PAN-inducted $24 \mathrm{~h}$, TRPC6 was not continuity distribution along the cell membrane, increased in the cytoplasm; (h) after PAN-inducted $48 \mathrm{~h}$, TRPC6 distribution increased in the cell membrane, part of the cell membrane lost, gathered into a granular, widely distributed in the cytoplasm; (c) PAN-inducted + DEX-treated 8 h; (f) PAN-inducted + DEX-treated 24 h; (i) PAN-inducted + DEX-treated 48 h, TRPC6 was more homogeneous distribution in the membrane at each time point.

\section{Disclosure}

The authors declare that they are coauthors and contributed equally to this work.

\section{Conflict of Interests}

The authors declare that they have no cinflict of interests.

\section{Acknowledgments}

This work was supported by the Natural Science Foundation Project of Guangdong Province (Project no. 5000424), the Technical Project of Guangdong Province (no. 2005B36001019), the Science and Technology Research
Project of Guangzhou (no. 2006Z3-E0231). The authors thank Professor Peter Mundel (America) and Professor Jie Ding, (Peking University First Hospital) for the Podocyte Clone. They thank the Central Laboratory of Guangzhou First Municipal Hospital for Technique Helps.

\section{References}

[1] K. Zandi-Nejad, A. A. Eddy, R. J. Glassock, and B. M. Brenner, "Why is proteinuria an ominous biomarker of progressive kidney disease?” Kidney International, Supplement, vol. 66, no. 92, pp. S76-S89, 2004.

[2] J. Reiser, K. R. Polu, C. C. Möller et al., "TRPC6 is a glomerular slit diaphragm-associated channel required for normal renal function," Nature Genetics, vol. 37, no. 7, pp. 739-744, 2005. 
[3] P. Mundel, J. Reiser, A. Z. M. Borja et al., "Rearrangements of the cytoskeleton and cell contacts induce process formation during differentiation of conditionally immortalized mouse podocyte cell lines," Experimental Cell Research, vol. 236, no. 1, pp. 248-258, 1997.

[4] C. Montell, "The TRP superfamily of cation channels," Science's STKE, vol. 2005, no. 272, p. re3, 2005.

[5] I. S. Ramsey, M. Delling, and D. E. Clapham, "An introduction to TRP channels," Annual Review of Physiology, vol. 68, pp. 619-647, 2006.

[6] A. Dietrich, H. Kalwa, B. R. Rost, and T. Gudermann, "The diacylgylcerol-sensitive TRPC3/6/7 subfamily of cation channels: functional characterization and physiological relevance," Pflugers Archiv European Journal of Physiology, vol. 451, no. 1, pp. 72-80, 2005.

[7] M. P. Winn, P. J. Conlon, K. L. Lynn et al., "Medicine: a mutation in the TRPC6 cation channel causes familial focal segmental glomerulosclerosis," Science, vol. 308, no. 5729, pp. 1801-1804, 2005.

[8] J. Reiser, K. R. Polu, C. C. Möller et al., “TRPC6 is a glomerular slit diaphragm-associated channel required for normal renal function," Nature Genetics, vol. 37, no. 7, pp. 739-744, 2005.

[9] C. C. Möller, C. Wei, M. M. Altintas et al., "Induction of TRPC6 channel in acquired forms of proteinuric kidney disease," Journal of the American Society of Nephrology, vol. 18, no. 1, pp. 29-36, 2007.

[10] T. Wada, J. W. Pippin, C. B. Marshall, S. V. Griffin, and S. J. Shankland, "Dexamethasone prevents podocyte apoptosis induced by puromycin aminonucleoside: role of p53 and Bcl2-related family proteins," Journal of the American Society of Nephrology, vol. 16, no. 9, pp. 2615-2625, 2005.

[11] T. Wada, J. W. Pippin, M. Nangaku, and S. J. Shankland, "Dexamethasone's prosurvival benefits in podocytes require extracellular signal-regulated kinase phosphorylation," Nephron, Experimental Nephrology, vol. 109, no. 1, pp. e8-e19, 2008.

[12] X. Sun, Z. Fang, Z. Zhu, X. Yang, F. He, and C. Zhang, "Effect of down-regulation of TRPC6 on the puromycin aminonucleoside-induced apoptosis of mouse podocytes," Journal of Huazhong University of Science and Technology Medical Science, vol. 29, no. 4, pp. 417-422, 2009.

[13] Y. H. Kim, M. Goyal, D. Kurnit et al., "Podocyte depletion and glomerulosclerosis have a direct relationship in the PANtreated rat," Kidney International, vol. 60, no. 3, pp. 957-968, 2001.

[14] C. X. Zheng, Z. H. Chen, C. H. Zeng, W. S. Qin, L. S. Li, and Z. H. Liu, "Triptolide protects podocytes from puromycin aminonucleoside induced injury in vivo and in vitro," Kidney International, vol. 74, no. 5, pp. 596-612, 2008.

[15] S. F. Liu, J. Ding, Q. F. Fan, and H. Zhang, "Establishment of a podocyte cell injury model induced by puromycin aminonucleoside," Beijing Da Xue Xue Bao, vol. 40, no. 6, pp. 586-589, 2008.

[16] W. Kriz, "TRPC6 - A new podocyte gene involved in focal segmental glomerulosclerosis," Trends in Molecular Medicine, vol. 11, no. 12, pp. 527-530, 2005.

[17] M. Estacion, W. G. Sinkins, S. W. Jones, M. A. B. Applegate, and W. P. Schilling, "Human TRPC6 expressed in HEK 293 cells forms non-selective cation channels with limited $\mathrm{Ca}^{2+}$ permeability," Journal of Physiology, vol. 572, no. 2, pp. 359377, 2006.

[18] H. Zhang, J. Ding, Q. Fan, and S. Liu, "TRPC6 up-regulation in Ang II-induced podocyte apoptosis might result from ERK activation and NF- $\kappa \mathrm{B}$ translocation," Experimental Biology and Medicine, vol. 234, no. 9, pp. 1029-1036, 2009.
[19] C. C. Möller, C. Wei, M. M. Altintas et al., "Induction of TRPC6 channel in acquired forms of proteinuric kidney disease," Journal of the American Society of Nephrology, vol. 18, no. 1, pp. 29-36, 2007.

[20] T. Wada, J. W. Pippin, C. B. Marshall, S. V. Griffin, and S. J. Shankland, "Dexamethasone prevents podocyte apoptosis induced by puromycin aminonucleoside: role of p53 and Bcl2-related family proteins," Journal of the American Society of Nephrology, vol. 16, no. 9, pp. 2615-2625, 2005.

[21] S. F. Liu, J. Ding, Q. F. Fan, and H. Zhang, "Establishment of a podocyte cell injury model induced by puromycin aminonucleoside," Beijing Da Xue Xue Bao, vol. 40, no. 6, pp. 586-589, 2008. 


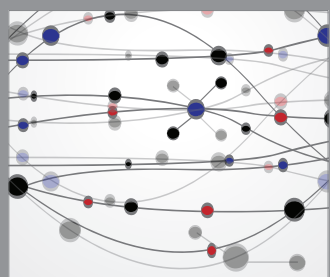

The Scientific World Journal
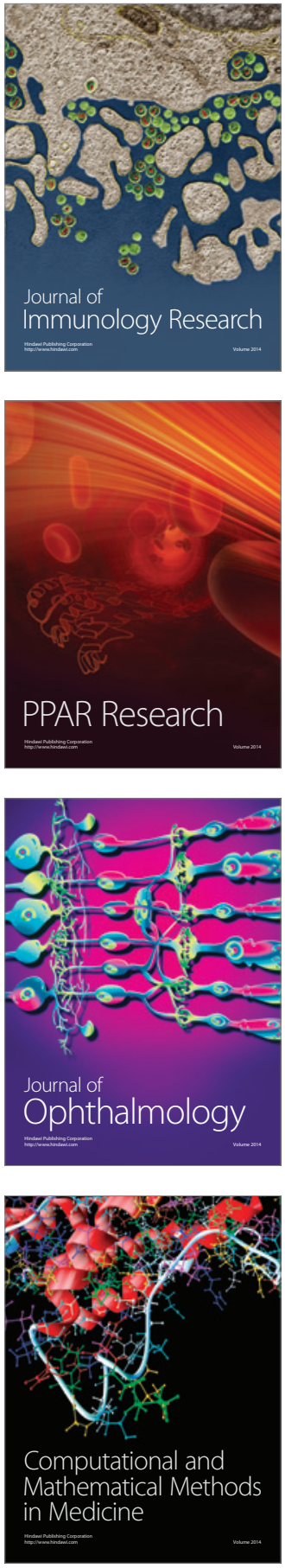

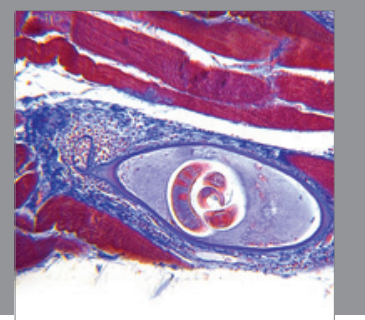

Gastroenterology

Research and Practice
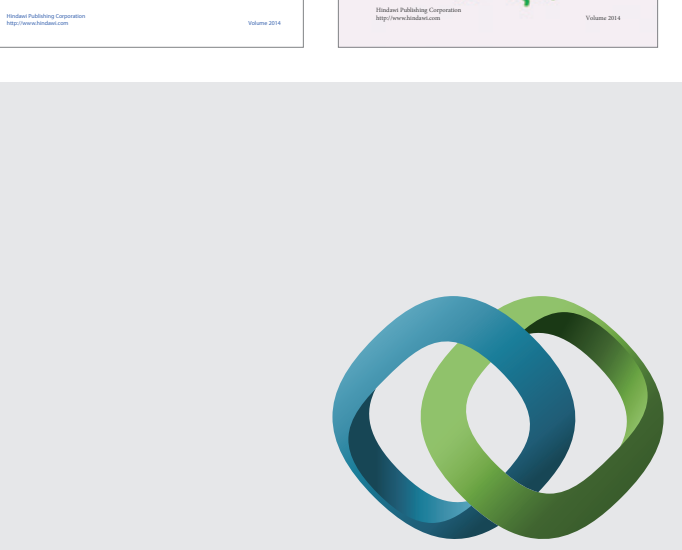

\section{Hindawi}

Submit your manuscripts at

http://www.hindawi.com
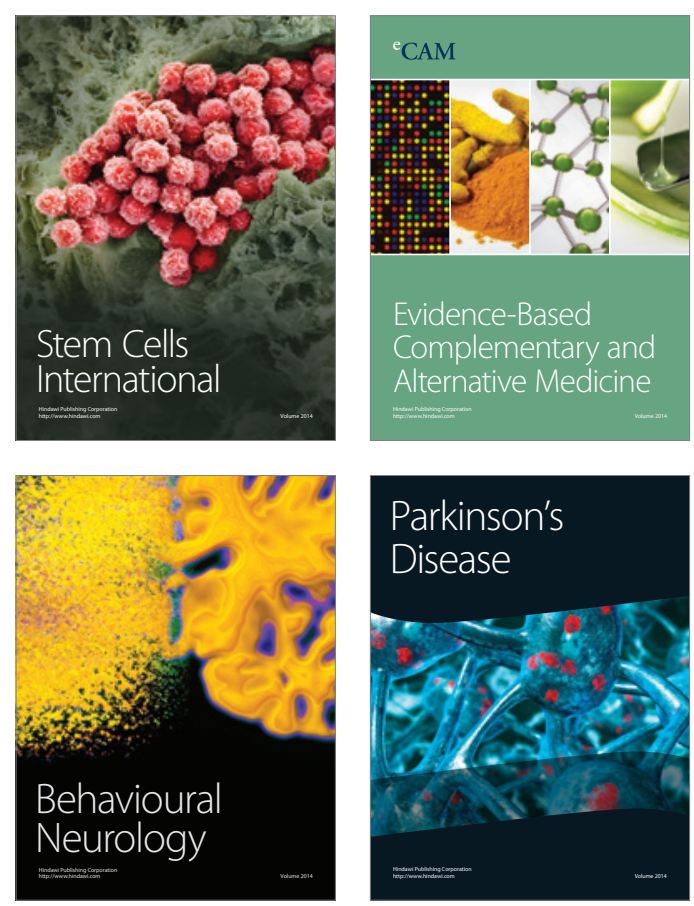

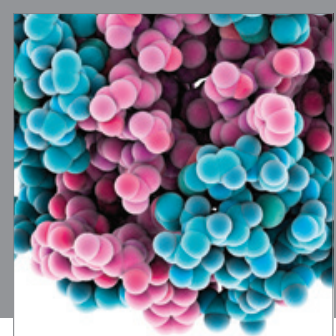

Journal of
Diabetes Research

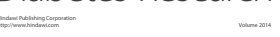

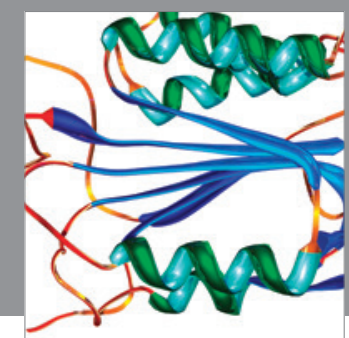

Disease Markers
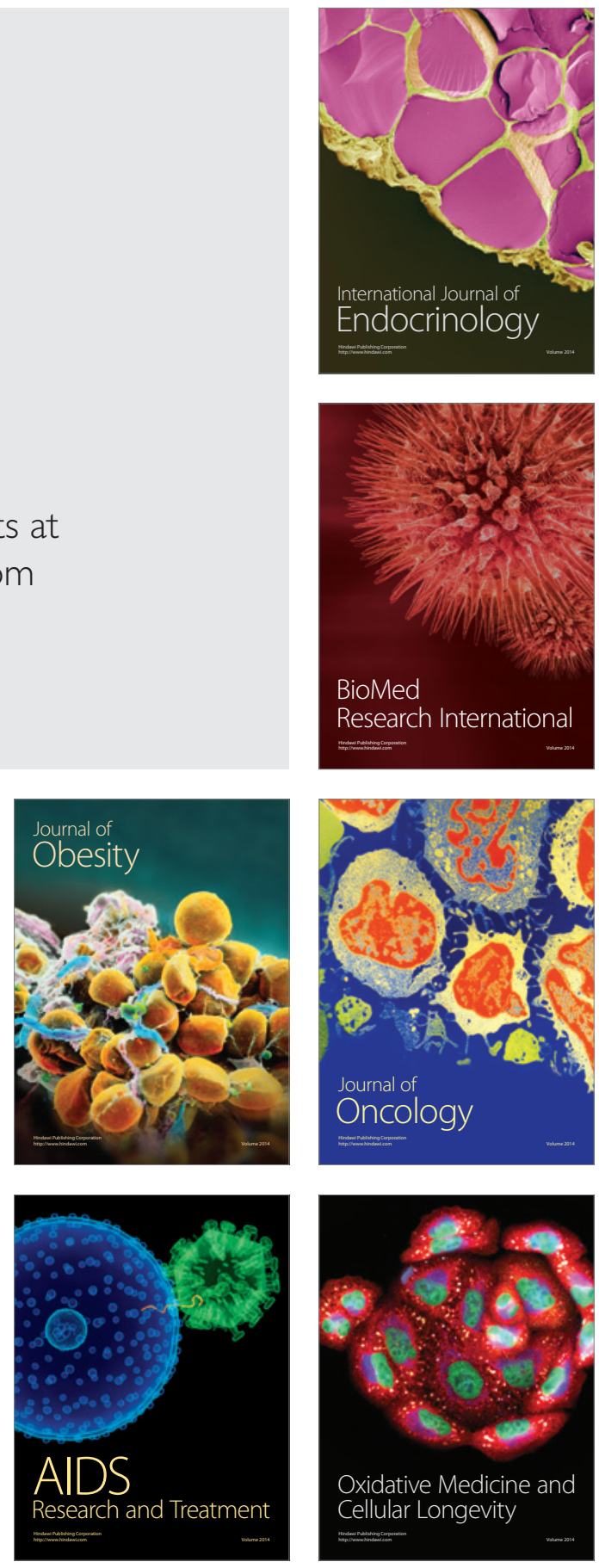